\title{
Effects of visual and auditory noise on visual choice reaction time in a continuous-flow paradigm
}

\author{
E. J. STOFFELS \\ Free University, Amsterdam, The Netherlands \\ and \\ M. W. VAN DER MOLEN \\ University of Amsterdam, Amsterdam, The Netherlands
}

\begin{abstract}
Two experiments were employed to investigate the processing speed of a visual target surrounded by visual noise (e.g., HHSHH or SSSSS) and auditory location cues (e.g., a left- or right-positioned tone) in a binary choice reaction time (RT) task. Visual noise impaired processing speed when it was incongruent with the target (e.g., HHSHH and SSHSS). Irrelevant location cues impaired reaction speed when the location of the tone did not correspond with the location of the correct response. When the visual stimuli were characters, the visual and auditory noise sources made additive contributions to mean visual choice RT. It was concluded therefore that visual congruity and location correspondence affect different process elements, response activation and response selection, respectively. This conclusion must be qualified, however, in that when the visual stimuli were left- or right-pointing arrows (e.g., $\leftarrow \leftarrow \leftarrow \leftarrow \leftarrow$ or $\leftarrow \leftarrow \rightarrow \leftarrow \leftarrow$ ), congruity and correspondence interacted. This interaction is interpreted to suggest that when visual and auditory noise are the same type (i.e., eliciting a left or right association) a cross talk between visual and auditory feature-detection channels might occur to produce a perceptual conflict on corresponding trials.
\end{abstract}

There are two extreme views of human information processing: continuous-flow models of information processing, which are based on the notion that the output of any process element is continuously available to all subsequent or concurrent processes, and serial discrete stage models, which are based on the assumption that output from a particular processing stage is available only after its predecessor has completed its information transformation. The former view implies that the partial results of process A can serve as input to process B before process A is completed. The continuous-flow conception of C. W. Eriksen and Schultz (1979), for example, assumes that information about the percept accumulates over time, and that as it accumulates, responses are continuously primed or activated. When the priming of a response reaches the evocation threshold, the response is executed.

Alternatives to the continuous-flow conception assume that reaction time (RT) consists of the sum of durations of elementary processors (i.e., stages) that operate serially. Accordingly, the output of stage A serves as the input for stage B, and stage B cannot begin until processing of stage $A$ is completed. A process is completed

This study was supported by a grant to the second author from the Society for Pure Research in The Netherlands (ZWO, Grant 153209). Helpful discussions with Michael G. H. Coles and J. Richard Simon are gratefully acknowledged. The first author's present address is Department of Psychology, University of Amsterdam Weesperplein 8, 1018 XA Amsterdam, The Netherlands. whenever its output satisfies criteria set for the subsequent stage (i.e., there is constant-stage output). The additivefactor method (AFM) is used to infer stages by determining the mutual relations between the effects of task variables on RT. Additive effects are interpreted to suggest that variables affect different stages, whereas interactions mean that variables affect a common stage (e.g., Sanders, 1980; Sternberg, 1969).

A main feature of serial-stage models is a discrete decision stage between input and responses. In AFM studies, the duration of this stage is typically manipulated by using stimulus-response (S-R) compatibility as a task variable. S-R compatibility refers to the degree of natural or overlearned relations between stimuli and responses. Although there are many types of compatibility, symbolic and spatial compatibility are perhaps the most common. Symbolic compatibility results from a relation between stimulus and response codes that simplifies the translation process, a left- or right-pointing arrow signaling a left or right buttonpress rather than vice versa, for example (see, e.g., Stoffels, van der Molen, \& Keuss, 1985, Experiment 1). Spatial compatibility results from a relation between the position of the stimulus and response key, a left or right light associated with a right- or leftpositioned response key, for example (see, e.g., Stoffels et al., 1985, Experiment 2). The AFM literature consistently reports additive effects of S-R compatibility and typical input variables, such as signal quality and signal discriminability, and typical output variables, such as 
response specificity, time uncertainty, and instructed muscle tension (see review by Sanders, 1980). According to the additive-factor logic, this data pattern suggests the existence of an independent central decision stage, which may be labeled the "response selection stage."

In contrast to serial-stage models, continuous-flow models pay little attention to response selection. Rather, responses in the continuous-flow model are elicited whenever one of the response channels is activated at a criterion level. Thus, responses can be evoked at different levels of percept development, depending upon the preset criterion. Stimulus congruity is the typical task variable used in the continuous-flow context. Consider, for example, the C. W. Eriksen and Schultz (1979) paradigm, in which subjects are required to respond to the central character of a five-character array. There are two possible characters (e.g., $\mathrm{H}$ or $\mathrm{S}$ ), and each signals a different response (e.g., a lever movement to the left or right). Two classes of stimulus arrays are presented, congruent and incongruent. In a congruent array, the central character is flanked by identical characters (e.g., HHHHH); in the incongruent array, it is flanked by characters that are designated to the alternative response (e.g., SSHSS). Thus, in both cases, the flankers are task-relevant. It is found consistently that RT is appreciably longer with incongruent arrays and that more incorrect responses are made to them. According to C. W. Eriksen and Schultz, the increases in RT and errors are due to the activation of correct and incorrect responses. The responses compete for execution so that the correct response is inhibited and its execution is delayed.

The use of S-R compatibility in AFM studies and of stimulus congruity in continuous-flow models seems to reflect the differential emphasis given to response selection and response activation, respectively. Sanders's (1980) review, for example, does not contain studies in which noise is systematically manipulated. To date, however, a number of studies have been conducted which have assessed auditory noise effects on visual RT, using AFM techniques. In these studies, subjects are required to press a left or right key in response to a visual stimulus. At stimulus onset, task-irrelevant auditory noise is presented to either the left or the right ear. RTs are little affected if the location of the noise and the correct response correspond (e.g., left ear and left keypress or right ear and right keypress). However, when the locations of the noise and correct response do not correspond (e.g., left ear and right keypress or right ear and left keypress), RTs are prolonged by $20-40 \mathrm{msec}$ and more incorrect responses are made. The effect of location correspondence is interpreted as evidence that the subject cannot attend solely to the visual stimulus and that the location of the noise elicits a natural tendency to react to the source of the auditory stimulation. It is the necessity to override this tendency that is assumed to account for the delay found on noncorresponding trials (see, e.g., Simon, 1970). AFM studies investigating the effect of the auditory noise on visual information processing report con- sistently additive combinations with stimulus quality and interactions with S-R compatibility (Acosta \& Simon, 1976; Simon \& Pouraghabagher, 1978; Simon, Sly, \& Vilapakkam, 1981; Stoffels et al., 1985). These results are taken to suggest that location cues associated with auditory noise interfere with the processing of the visual stimulus at the response-selection stage, not with the encoding of the visual stimulus.

Stimulus congruity and location correspondence might be considered as experimental analogues. Superficially, at least, the phenomena resemble each other. From a continuous-flow perspective, the elevated RTs observed on noncorresponding trials are due to the activation of both the correct response-elicited by the visual stimulus-and the incorrect response-evoked by the location of the auditory noise. The responses compete with each other so that the correct response is inhibited and delayed in its execution. If this is true, one would expec that a factorial combination of stimulus congruity and S-F compatibility would yield a data pattern identical to that resulting from the combination of location correspondence and S-R compatibility.

Only recently, however, Bashore and Osman (1987) investigated the effects of the combination of stimulus congruity and S-R compatibility in an Eriksen task. Preliminary results showed the prolongation of RT typical for incongruent and incompatible trials. Additionally, it was found that the cost of incompatibility was somewha reduced when an incompatible response was made to an incongruent array relative to a congruent array (32 vs. $40 \mathrm{msec}$, respectively). Interestingly, P300 latency-. positive shift of a stimulus-locked brain potential that $i$. assumed to reflect the completion of the stimulus evaluation process (e.g., McCarthy \& Donchin, 1981)-was alsc increased by incompatibility and incongruity, but the mag. nitude of the effects differed. Although the effect of stimu. lus congruity was comparable for RT and P300, the ef fect of S-R compatibility was much less on P300 than or RT. Finally, Bashore and Osman observed that N200-: negative deflection of the brain potential-was unaffecter by congruity but was prolonged substantially by compati bility. According to Bashore and Osman, this data pat tern suggests that congruity and compatibility may in fluence different process elements. It should be noted however, that previous psychophysiological investigation have found the congruity effect to be almost as large o1 response-related processes as on stimulus-relater processes (Coles, Gratton, Bashore, C. W. Eriksen, Donchin, 1985). In any event, it is important to note th: the relative independence of stimulus congruity as a func tion of S-R compatibility suggests that congruity and com patibility influence different response-related processe: substantiating the different perspectives of the discret serial-stage and continuous-flow models.

The aim of the present study was to further assess thi hypothesis by factorially combining stimulus congruit and location correspondence. Two experiments were con ducted in which subjects were required to respond to 
visual target by pressing a left or right key. The targets could be flanked by identical symbols (congruence), by symbols signaling the incorrect response (incongruence), or by task-irrelevant symbols that shared features with both task-relevant symbols (orthogonal). The onset of the visual stimulus array was accompanied by auditory noise presented to the ear ipsilateral to the correct response (correspondence), to the ear contralateral to the correct response (noncorrespondence), or to both ears (neutral). Two different experimental designs were used. The presentations of visual arrays could be either blocked (i.e., congruent, incongruent, or orthogonal arrays were presented between blocks of trials) or mixed (i.e., all three arrays were presented within blocks of trials). Coles et al. (1985) showed that a blocked design led to faster stimulus evaluation on all trials, as indexed by shorter P300 latencies and shorter RTs, and that the effect on RT was more pronounced on congruent trials than on incongruent trials. Assuming a resemblance between stimulus congruity and location correspondence, the design (blocked vs. mixed) manipulation should not, if their resemblance has a perceptual origin, affect the difference in RT between corresponding and noncorresponding trials. In contrast, if both variables affect identical response-related elements, the experimental design should differentially affect response speed on corresponding and noncorresponding trials. The two experiments reported here were identical, except that in Experiment 1 the visual array consisted of characters, as in the Coles et al. (1985) study, and in Experiment 2 the visual array consisted of left- or rightpointing arrows, as in the Bashore and Osman (1987) and the Stoffels et al. (1985) studies.

\section{METHOD}

\section{Subjects}

The subjects were 36 dental students between 20 and 25 years of age. All reported normal vision and hearing. They were paid a fixed amount for their participation.

\section{Apparatus and Event Sequences}

There were two sets of stimulus arrays (viz., characters and arrows). For the characters, the target character was either an S or an $\mathrm{H}$. The target was surrounded by four task-relevant flankers (Hs or Ss) or by four task-irrelevant flankers (Bs). The task-relevant flankers were either congruent or incongruent. The task-irrelevant flankers were orthogonal, meaning that no response was associated with those flankers. For the arrows, the target was either a leftor a right-pointing arrow. The target was accompanied by arrows pointing in the same direction as the target (congruent), in the opposite direction (incongruent), or upwards (orthogonal). Figure 1 shows the sets of character and arrow arrays. The stimulus array was $12.5 \mathrm{~cm}$ long and $2 \mathrm{~cm}$ high. The subject was seated $160 \mathrm{~cm}$ from the screen (i.e., effecting a $7.3^{\circ}$ visual angle). The luminance of the array was $40 \mathrm{~cd} / \mathrm{m}^{2}$. The exact position of the target was marked by a vertical line above and a vertical line below the target. A Kodak slide projector was used for presentation of the stimulus array.

A trial started with a $3000-\mathrm{Hz} / 60-\mathrm{dB}$ warning stimulus (WS) that was on for $250 \mathrm{msec}$. The stimulus was presented $2,000 \mathrm{msec}$ after WS offset. The visual stimulus was accompanied by a binaural or a monaural (left or right) tone of $1000 \mathrm{~Hz} / 80 \mathrm{~dB}(\mathrm{~A})$. The loca- tion cue associated with the monaural tone could either correspond with the location of the keypress response to the visual stimulus (corresponding trials) or not (noncorresponding trials). The binaural tone did not constitute a left or right association and served as a neutral reference. The total trial duration was $5 \mathrm{sec}$. Event-sequence timing and latency recording were controlled and registered by a PDP-11/04 minicomputer.

\section{Procedure}

The subject was seated in a comfortable chair in a dimly lit and sound-attenuated room. Two response keys were mounted on a table in front of the subject. One group of 20 subjects received the character set (Experiment 1), and another group of 16 subjects received the arrow set (Experiment 2). The within-subjects factors were design ( 2 ; blocked vs. mixed presentation of the visual noise), visual congruity ( 3 ; congruent, incongruent, or orthogonal trials), and location correspondence (3; corresponding, noncorresponding, or neutral trials). There were two sessions-one blocked and one mixed-each of which consisted of three trial blocks. For the mixed session, there were 18 unique trials (stimulus array [6] $\times$ location [3]) within a block; each was presented five times. This yielded 30 replications of a particular visual noise $\times$ location correspondence combination over three blocks, with replications collapsed over the responding hands. For the blocked session, the congruent, incongruent, and orthogonal stimulus arrays were each presented in a different block of trials. The location cue was again randomly presented within a block. Half of the group of subjects began with the mixed session; the other half began with the blocked sessions. The presentation order of the three blocks in the blocked sessions was counterbalanced across subjects. There were 15 warm-up trials before each trial block. The day before the test session, the subjects received six blocks of practice trials. Accuracy, reaction speed, and regular performance were monitored in terms of errors, mean RT, and standard deviation, respectively.

\section{RESULTS}

Separate analyses of variance (Finn, 1978) were performed on binaural and monaural trials. Preplanned comparisons were carried out to assess the effects of visual noise, with orthogonal and neutral noise as references.

\section{Experiment 1}

Binaural trials. The analysis performed on binaural or neutral trials comprised 2 within-subjects factors: design (blocked vs. mixed) and visual congruity (congruent, incongruent, and orthogonal). The subjects performed faster in the blocked design $(370 \mathrm{msec})$ than in the mixed design $(391 \mathrm{msec})[F(1,19)=19.51, p<.01]$. The RTs for the congruent trials were faster $[348 \mathrm{msec}, F(1,19)$ $=64.42, p<.01]$ and the RTs for incongruent trials were slower $[412 \mathrm{msec}, F(1,19)=73.07, p<.01]$ than the RTs for orthogonal trials $(381 \mathrm{msec})$. The difference in mean RT between congruent and orthogonal trials was larger for the blocked design ( $42 \mathrm{msec}$ ) than for the mixed design $(24 \mathrm{msec})[F(1,19)=4.86, p<.04]$. This difference did not exist between incongruent and orthogonal trials [32 vs. $29 \mathrm{msec}, F(1,19)<1$, n.s.]. The effects of congruity and design for the neutral $(N)$ trials are illustrated in the two leftmost panels of Figure 2.

Monaural trials. This analysis comprised 3 withinsubjects factors: design (blocked vs. mixed), visual congruity (congruent, incongruent, and orthogonal trials), and 


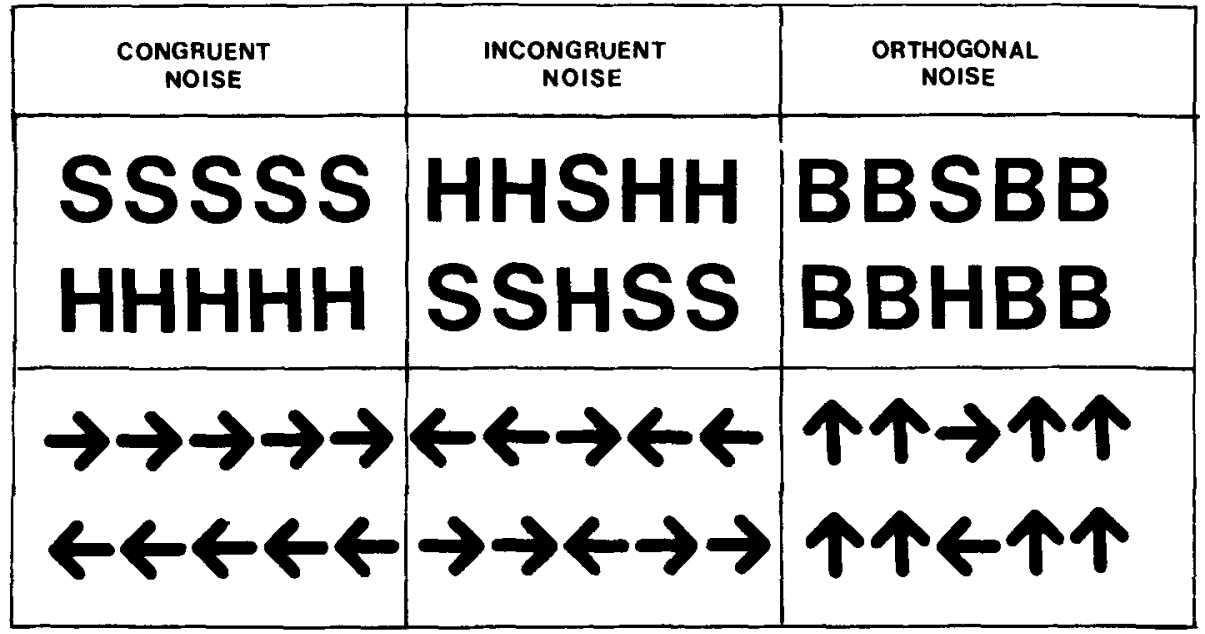

Figure 1. Stimuli used in Experiments 1 and 2. The upper row depicts the character arrays, and the lower row depicts the arrow arrays. The left panels show the congruent stimulus arrays, the middle panels show the incongruent arrays, and the right panels show the orthogonal arrays. The center symbol of the arrays served as the visual reaction stimulus.

location correspondence (corresponding vs. noncorresponding trials). RTs were faster in the blocked design than in the mixed design [ 371 vs. $391 \mathrm{msec}, F(1,19)=$ $19.30, p<.01]$. RTs for the congruent trials were faster [351 msec, $F(1,19)=51.65, p<.01]$ and RTs for incongruent trials were slower $[407$ msec, $F(1,19)=69.43$, $p<.01]$ than were RTs for orthogonal trials $(382 \mathrm{msec}$ ).
The effect of visual congruity was larger in the blocked sessions than in the mixed sessions, but only when the congruent arrays are contrasted with the incongruent arrays $[69$ vs. $50 \mathrm{msec}, F(1,19)=8.85, p<.01]$. Corresponding trials were faster $(374 \mathrm{msec})$ than noncorresponding trials $[388 \mathrm{msec}, F(1,19)=52.44, p<.01]$. Importantly, the effect of location correspondence did not

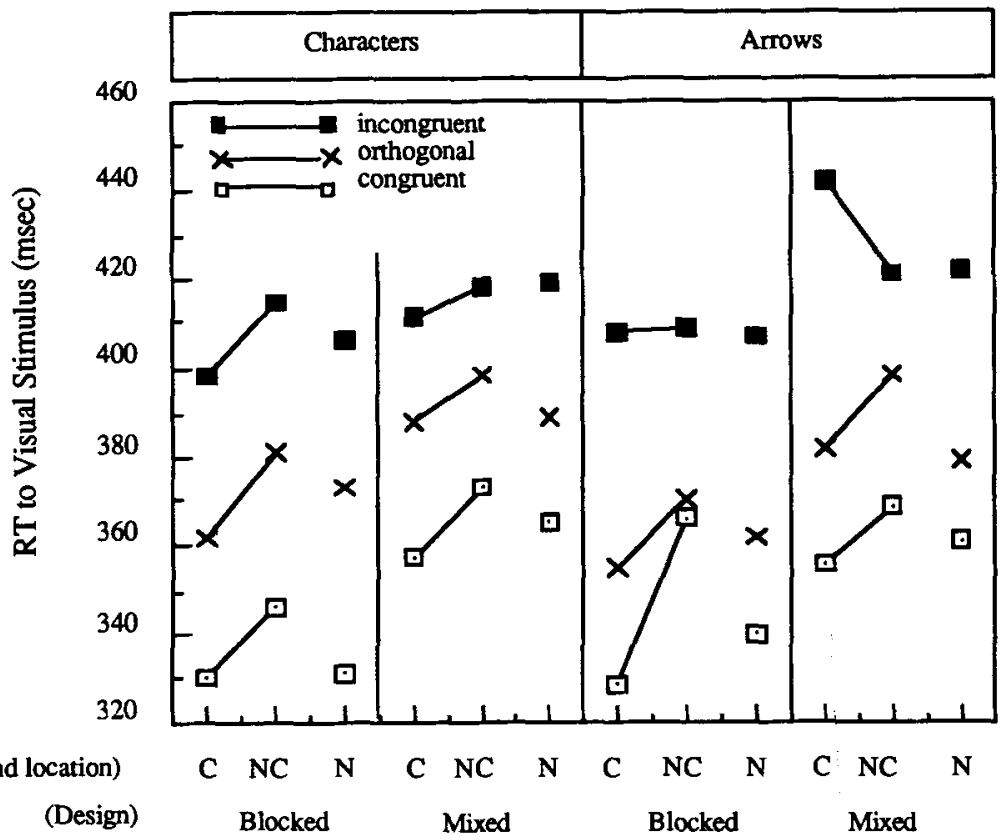

Figure 2. Mean reaction times (RTs) in milliseconds for each design $\times$ visual congruity $\times$ location correspondence condition. C denotes the corresponding trials, NC denotes the noncorresponding trials, and $\mathbf{N}$ denotes the neutral (binaural) trials. The two panels on the left show the mean RTs for Experiment 1, and the two panels on the right show the mean RTs for Experiment 2. The blocked conditions are depicted to the left of the mixed conditions. 
vary across congruity and blocking conditions. The results for monaural trials are depicted in the two leftmost panels of Figure 2.

\section{Experiment 2}

Binaural trials. The performance of subjects was faster in the blocked design ( $370 \mathrm{msec}$ ) than in the mixed design $(388 \mathrm{msec})[F(1,15)=16.30, p<.01]$. The RTs for congruent trials were faster $[351 \mathrm{msec}, F(1,15)=$ $46.82, p<.01]$ and the RTs for incongruent trials were slower $[415 \mathrm{msec}, F(1,15)=104.17, p<.01]$ than the RTs for the orthogonal trials $(371 \mathrm{msec})$. This pattern holds for both the blocked and the mixed designs. The rightmost panels of Figure 2 depict the congruity and design effects on RT to the arrow arrays for the $\mathrm{N}$ trials.

Monaural trials. The effects of location correspondence as a function of congruity and design are depicted in the two rightmost panels of Figure 2. These panels show a highly complex data pattern. First, in the orthogonal conditions, the effect of location correspondence does not discriminate between the blocked $(15 \mathrm{msec})$ and the mixed $(14 \mathrm{msec})$ design $[F(1,15)<1]$. Note that the magnitude of this effect is almost the same as in the first experiment. In contrast to the first experiment, however, there was a marked difference between congruent and incongruent trials and between blocked and mixed designs. The effect of location correspondence on congruent trials was much stronger in the blocked condition $(36 \mathrm{msec})$ than in the mixed condition $(14 \mathrm{msec})[F(1,15)=25.03$, $p<.01]$. On incongruent trials, however, the difference between noncorresponding and corresponding trials in the blocked condition was effectively zero, whereas in the mixed condition, corresponding trials were even slower than noncorresponding trials $[-20 \mathrm{msec}, F(1,15)=9.47$, $p<.01]$. Finally, planned comparisons indicated that, in the blocked design, the effect of location correspondence was smaller on orthogonal trials than on congruent trials $[F(1,15)=19.77, p<.01]$; the effect on incongruent trials did not differ from that on orthogonal trials $[F(1,15)=1.86$, n.s. $]$. In contrast, in the mixed design, the effect of location correspondence on orthogonal trials was similar to that on congruent trials $[F(1,15)<1]$, but smaller than that on incongruent trials $[F(1,15)=32.29$, $p<.01]$.

Errors. Table 1 shows the error rates for both the character arrays of Experiment 1 (left) and the arrow arrays of Experiment 2 (right) as a function of congruity and location correspondence. Overall, error rate was low. More errors were made on corresponding trials than on noncorresponding trials. The effect of congruity did not systematically affect accuracy.

\section{DISCUSSION}

The primary purpose of the present research was to assess the combined effects of visual and auditory noise on visual information processing in a task developed by C. W. Eriksen and his colleagues (e.g., B. A. Eriksen \& C. W. Eriksen, 1974; C. W. Eriksen \& Schultz, 1979).

Before discussing any conclusions, however, we need first to establish that auditory stimulation per se did not change the nature of the Eriksen task. The results obtained on binaural trials allow an independent assessment of visual congruity effects, since no left or right location cues are associated with noise presented to both ears (see Simon, 1982). The binaural data pattern is highly consistent for the two experiments. The visual congruity effect was $65 \mathrm{msec}$ for character arrays and $64 \mathrm{msec}$ for arrow arrays. These results are comparable with the 50- to 100msec effects of visual congruity obtained in previous studies (cf. Bashore \& Osman, 1987). Additionally, the effect of the design manipulation (i.e., blocked vs. mixed presentation of visual noise) is the same for the two experiments and replicates the design effect reported by Coles et al. (1985). The blocked presentation of noise accelerated response speed for all noise levels (21 msec for character arrays and $18 \mathrm{msec}$ for arrow arrays). As in the Coles et al. study, the gain was more pronounced for congruent than for incongruent arrays. In the continuous-flow context, the visual congruity effect is interpreted in terms of stimulus evaluation and response competition. Incongruent noise produces conflict in stimulus evaluation, which slows the evaluation process and activates both response channels, which in turn results in response competition. The blocking of visual noise speeds up the evaluation process for both congruent and incongruent noise and lowers the subject's response criterion when the noise is congruent (cf. Coles et al., 1985, p. 545-546). Coles et al. suggested that the blocking of visual noise facilitated the stimulus evaluation process because localization of the central target is not necessary; on congruent trials, all elements of the array are the same; on incongruent trials, the flankers are consistently different from the central target. The current experiment, however, included

Table 1

Percentage Errors for Character and Arrow Stimuli

\begin{tabular}{|c|c|c|c|c|c|c|c|c|c|c|c|c|}
\hline \multirow[b]{3}{*}{ Noise } & \multicolumn{6}{|c|}{ Characters } & \multicolumn{6}{|c|}{ Arrows } \\
\hline & \multicolumn{3}{|c|}{ Blocked } & \multicolumn{3}{|c|}{ Mixed } & \multicolumn{3}{|c|}{ Blocked } & \multicolumn{3}{|c|}{ Mixed } \\
\hline & $\mathrm{C}$ & $\mathrm{NC}$ & $\overline{\mathbf{N}}$ & $\mathrm{C}$ & $\mathrm{NC}$ & $\overline{\mathbf{N}}$ & $\vec{C}$ & $\mathrm{NC}$ & $\overline{\mathbf{N}}$ & $\bar{C}$ & $\mathrm{NC}$ & $\overline{\mathbf{N}}$ \\
\hline Incongruent & 3.3 & 2.9 & 2.5 & 4.1 & 5.0 & 4.1 & 2.7 & 1.7 & 1.6 & 3.9 & 2.9 & 2.9 \\
\hline Orthogonal & 2.5 & 1.8 & 2.4 & 1.5 & 2.6 & 1.4 & 1.3 & 1.7 & 1.0 & 1.7 & 1.3 & 0.7 \\
\hline Congruent & 0.8 & 2.1 & 1.5 & 0.5 & 0.5 & 0.5 & 1.5 & 2.6 & 1.8 & 0.5 & 0.7 & 0.9 \\
\hline
\end{tabular}

Note $-\mathrm{C}=$ corresponding, $\mathrm{NC}=$ noncorresponding, and $\mathrm{N}=$ neutral trials. 
an orthogonal noise condition in which the flankers did not give any clue about the central target. Yet, the effect of blocking did not discriminate between responses to orthogonal and incongruent noise arrays. This might indicate that the effect of blocking on stimulus evaluation actually consists of two effects: an effect at the level of feature analysis and an effect at the level of target localization. For congruent and incongruent noise levels, the effect of blocking is predominantly on target localization, whereas for orthogonal trials, the effect may consist primarily of a reduction in the time needed for feature analysis. In this respect, the current data provide additional support for Coles et al.'s idea that stimulus evaluation comprises two subprocesses: feature analysis and target localization.

Second, it is necessary to determine whether the presentation of visual noise per se has the qualitative effect on performance that is usually observed in tasks that confront the subject with location cues associated with taskirrelevant auditory noise. The orthogonal noise condition might provide a neutral baseline in that the flankers in the orthogonal noise condition do not differentially activate experimentally defined responses. The effect of location correspondence was consistent across experiments $-15 \mathrm{msec}$ for character arrays and $14 \mathrm{msec}$ for arrow arrays-and design conditions- $17 \mathrm{msec}$ for the blocked noise levels and $15 \mathrm{msec}$ for the mixed noise levels. The direction and magnitude of the present location correspondence effect is in the 10- to 50-msec range of the effects reported previously in the literature (see Ragot \& Lesevre, 1986). Although there are various interpretations of location correspondence (e.g., Whitaker, 1982), Simon's explanation is perhaps the most widely cited (Simon, 1969, 1982). He suggested that auditory noise elicits a natural tendency to respond toward the noise source, and that when the location of the noise source does not correspond with the location of the correct response this tendency must be inhibited. The delay observed on noncorresponding trials is due to the inhibition of the incorrect response tendency. It is important to note that as far as the orthogonal condition is concerned, the effects of location correspondence and design are additive. Assuming that the design effect on the processing of orthogonal arrays is on feature analysis, this result is consistent with the AFM finding of an additive relation between stimulus quality and location correspondence. Both results suggest that the effect of location correspondence does not interfere with visual information processing at an early perceptual level (e.g., Simon, 1982; Simon \& Pouraghabagher, 1978; Stoffels et al., 1985).

At this point, we may conclude that auditory noise per se does not change the pattern of results that is commonly observed when the level and presentation mode of visual noises is varied. Additionally, visual noise per se does not change the direction and magnitude of the effect of monaural noise on visual reaction time. But what happens when the two effects are combined? The current data indicate that there is no simple answer to this question. For character arrays, the effects of visual congruity and location correspondence were additive, but an interaction was observed for arrow arrays. Moreover, the design manipulation did not change the relation between the effects of visual congruity and location correspondence for character arrays, whereas a higher order interaction was observed for arrow arrays. The additive relation of visual congruity and location correspondence could be interpreted by taking visual congruity as a perceptual variable and location correspondence as a motor variable. Such an interpretation would be supported by the present findings of an interaction between visual congruity and the design manipulation and the lack of an interaction between location correspondence and design. It should be noted, however, that Coles et al. (1985) provided strong support for an interpretation of visual congruity in terms of both perceptual (stimulus evaluation) and motor (response competition) processes. In this respect, a differentiation between the effects of visual congruity and location correspondence in terms of perceptual versus motor processors may be too simple. Moreover, what is so different between visual congruity and location correspondence? Superficially, C. W. Eriksen's interpretation of the effects of visual (congruity) noise and Simon's interpretation of the effect of auditory (location) noise are similar, in that both interpretations emphasize the contribution of response competition in the adverse effects of noise on the speed of information processing. Finally, the current difference between the character and arrow data patterns seems to prohibit a simple explanation.

In contrast to the character arrays, the noise induced by left- or right-pointing arrows interacts with the location cues associated with auditory noise. The interaction is markedly different for the blocked and mixed presentations of the visual noise. In the blocked condition, congruent visual noise is associated with a pronounced effect of location correspondence (about $40 \mathrm{msec}$ ). For incongruent noise arrays, however, the difference in RT between corresponding and noncorresponding trials seems to disappear. In the mixed condition, the pronounced effect of location correspondence combined with congruent visual noise arrays is reduced to normal proportions; that is, its magnitude is the same as that for the orthogonal arrays. Most surprisingly, for incongruent visual noise arrays, response speed was slower on corresponding than on noncorresponding trials.

The heterogeneous data pattern for arrow arrays cannot be rejected as an aberration. The results obtained when the presentation of visual noise was blocked are consistent with the findings commonly observed in AFM studies investigating the effect of location correspondence as a function of symbolic S-R compatibility (e.g., Simon et al., 1981, Experiment 3; Stoffels et al., 1985, Experiment 1). In the AFM context, the disappearance of the difference in RT between corresponding and noncorresponding trials is usually interpreted in terms of a suppression due to a higher task load on the response-selection mechanism. A reversal of the location correspondence ef- 
fect has been previously observed in AFM studies manipulating spatial S-R compatibility (e.g., Hedge \& Marsh, 1975; Simon et al., 1981, Experiments 1 and 2). Hedge and Marsh explained the reversal of the location correspondence effect in terms of a notion that they called logical recoding:

For a given logical recoding (identity or reversal) of the relevant stimulus attribute (color), responding was faster for trials in which the recoding of the irrelevant stimulus attribute (position) was of the same logical type as that of the relevant stimulus attribute, than for trials in which the logical recoding of the irrelevant stimulus attribute was opposite in type. (Hedge \& Marsh, 1975, p. 435)

In other words, Hedge and Marsh suggested that when subjects have to translate a left-pointing stimulus into a right keypress, they also recode or reverse the irrelevant location cue associated with auditory noise so that they now respond faster on the side opposite to the location of the auditory noise. The logical recoding hypothesis, however, is difficult to reconcile with the results obtained for incongruent character arrays and for the incongruent arrow arrays presented in the blocked mode. Do subjects not recode when the stimulus is a letter, and do they not reverse when the noise is presented in blocked rather than in mixed series? Simon questioned the logical recoding hypothesis by suggesting that the reversal of the location correspondence effect obtained in S-R compatibility studies must be explained in terms of a confounding with a variety of spatial compatibility; he called this "displaycontrol arrangement correspondence" (Simon et al., 1981). The present results, however, clearly indicate that a reversal of the location correspondence effect can be obtained without changing the "display-control arrangement correspondence" (i.e., in all conditions of the present experiment, the visual stimulus was presented from a single central location).

We will turn now to the hypothesis we submitted in a previous study to explain the reversal of the location correspondence effect that occurs in S-R compatibility tasks (Stoffels et al., 1985). An elaborated version of this hypothesis may provide a unified account for the current findings. Previously, we interpreted the reversal of the location correspondence effect in terms of a complex interaction of two location cues:

In the compatible task, location cues were presented on the same side on corresponding trials and on opposite sides on non-corresponding trials. In contrast, in the incompatible task location cues were presented on the same side on noncorresponding trials and on opposite sides on corresponding trials. Assuming that reactions are faster when task relevant and irrelevant location cues are presented on the same side (left stimulus light and left ear stimulation or right stimulus light and right ear stimulation) than when location cues are presented on opposite sides (left stimulus light and right ear stimulation or right stimulus light and left ear stimulation) one might expect faster reactions on corresponding trials than on non-corresponding trials in the compatible task and the reverse in the incompatible task. (Stoffels et al., p. 60)
In other words, we suggested that the location cues associated with visual and auditory stimulation induce, using Ragot's (Ragot \& Lesevre, 1986) terminology here, a "cross talk" between visual and auditory featuredetection channels. According to this view, the reversal of the location correspondence effect in the present study results from a perceptual conflict between the location cues that are associated with monaural stimulation and the location cues that become available early in the stimulusevaluation process, which seems to be determined by the flankers rather than by the central target. The absence of a difference in RT between corresponding and noncorresponding trials for incongruent arrow arrays in the blocked condition might indicate that it is apparently easier for subjects to deal with the perceptual conflict when they know in advance what kind of visual noise will be presented. The exaggerated effect of location correspondence in the case of a blocked presentation of congruent arrow noise can be interpreted in terms of a strategy shift. In this condition, it is advantageous for the subject to respond as quickly as possible to the location cues provided by the arrow arrays. Obviously, the uninhibited tendency to respond to the location cues provided by the visual stimulus array enhanced the effect of the location cues associated with the monaural noise. ${ }^{1}$

In summary, our findings lead us to the following conclusions:

1. Visual letter noise (commonly used in Eriksen tasks) and monaural location noise (employed in AFM studies) are processed separately, that is, either sequentially or in parallel. Our data should not be interpreted to indicate that the perceptual analysis of the visual and auditory stimuli occurred in parallel. The evaluation of visual and auditory stimuli may very well have been sequential.

(a) The additive relation of the effects of visual congruity and location correspondence might suggest, contrary to the AFM logic, that both variables affect a common process-that is, response competition-so that the increase in RT to incongruent letter noise is simply added to the delay in response speed on noncorresponding auditory noise trials. We do not consider this hypothesis very plausible, however. If true, we would expect that the design manipulation-blocked versus mixed presentation of visual noise arrays-would change the effect of location correspondence, as it did the effect of visual congruity. Albeit unlikely, the hypothesis can be tested by a psychophysiological investigation employing electromyographic (EMG) measures. The EMG should differentiate not only between congruent and incongruent visual noise arrays, but also between corresponding and noncorresponding auditory noise trials.

(b) Alternatively, the additive relation of visual congruity and location correspondence might suggest that these variables affect different response-related process elements: response competition and response selection, respectively. This hypothesis is consistent with Schweickert and Boggs's (1984) interpretation of the results commonly observed in color-word Stroop tasks. In reviewing the literature on central limitations in information 
processing, they considered two hypotheses, one concerned with decision and one concerned with response conflict. As for the Stroop task, they cited evidence that both decision and response conflict were bottlenecks (Schweickert \& Boggs, p. 157). In other words, both hypotheses may be correct. That is, the delay produced by response conflict may have something to do with the problem of selecting the response channel out of the conflict set when several response channels are activated. The same reasoning might apply to the current doublestimulation task. In this case, a psychophysiological investigation might provide support by exclusion. That is, neither P300 nor the EMG should differentiate between corresponding and noncorresponding auditory noise trials.

2 . We suggest that the interaction between the effects of visual congruity and location correspondence in the arrow experiment reflects a cross talk between visual and auditory feature-detection channels. In this experiment, a perceptual cross talk could occur because the visual and auditory noise were of the same type, that is, both noise sources provided location cues. The cross talk between visual and auditory channels resulted in an apparent reversal of the location correspondence effects on incongruent visual noise trials in a mixed visual noise presentation. On corresponding trials, as opposed to noncorresponding trials, the conflict between visual and auditory location cues caused an extra time delay. A psychophysiological investigation should result in longer $\mathrm{P} 300$ latencies on corresponding/incongruent arrow arrays.

3. We consider the current observation of the additive relation between visual congruity and location correspondence effects as the most important result of the present research. We do not want to suggest that it provides support for the serial-stage conception of the information flow at the cost of continuous-flow models. What we do want to suggest, however, is that continuous-flow models should account for processes that may be labeled "response selection."

\section{REFERENCES}

Acosta, E., JR., \& Simon, J. R. (1976). The effect of irrelevant information on the stages of processing. Journal of Motor Behavior, 8 , 181-187.

Bashore, T. R., \& OSMAn, A. (1987, June). On the temporal relation between perceptual analysis and response selection: A psychophysiological investigation of stimulus congruency and $S-R$ compatibility effects on human information processing. Paper presented at the Fourth International Conference on Cognitive Neuroscience, Paris-Dourdan, France.

Coles, M. G. H., Gratton, G., Bashore, T. R., Eriksen, C. W., Donchin, E. (1985). A psychophysiological investigation of the continuous flow model of human information processing. Joumal of Experimental Psychology: Human Perception \& Performance, 11, 529-553.

ERIKSEN, B. A., \& ERIKSEN, C. W. (1974). Effect of noise letters upon the identification of a target letter in a nonsearch task. Perception \& Psychophysics, 16, 143-149.

Eriksen, C. W., Schultz, D. W. (1979). Information processing in visual search: A continuous flow conception and experimental results. Perception \& Psychophysics, 25, 249-263.
FinN, J. D. (1978). Multivariance: Univariate and multivariate analysis of variance, covariance, regression and repeated measures (Version VI, Release 2). Chicago: International Educational Services.

Hedge, A. , Marsh, N. W. A. (1975). The effect of irrelevant spatial correspondences on two-choice response-time. Acta Psychologica, 39, 427-439.

McCarthy, G., Donchin, E. (1981). A metric for thought: A comparison of P300 latency and reaction time. Science, 211, 77-80.

RAGOT, R., LESEVRE, N. (1986). Electrophysiological study of intrahemispheric S-R compatibility effects elicited by visual directional cues. Psychophysiology, 23, 19-27.

SANDERS, A. F. (1980). Stage analysis of reaction processes. In G. J. Stelmach \& J. Requin (Eds.), Tutorials in motor behavior. Amsterdam: North Holland.

SCHWEICKERT, R., BoGGs, G. J. (1984). Models of central capacity and concurrency. Joumal of Mathematical Psychology, 28, 223-281.

SimoN, J. R. (1970). Stereotypic reaction in information processing. In L. E. Smith (Ed.), Proceedings of the CIC Symposium on Psychology of Motor Learning. Chicago: Athletic Institute.

Simon, J. R. (1982). Effect of an auditory stimulus on the processing of a visual stimulus under single- and dual-task conditions. Acta Psychologica, 51, 61-73.

Simon, J. R., Pouraghabagher, A. R. (1978). The effect of aging on the stages of processing in a choice reaction time task. Journal of Gerontology, 33, 553-561.

Simon, J. R., Sly, P. E., \& Vilapakkam, S. (1981). Effect of compatibility of S-R mapping on reactions toward the stimulus source. Acta Psychologica, 47, 63-81.

STERNBERG, S. (1969). The discovery of processing stages: Extensions of Donders' method. Acta Psychologica, 30, 276-315.

Stoffels, E. J., van der Molen, M. W., \& Keuss, P. J. G. (1985). Intersensory facilitation and inhibition: Immediate arousal and location effects of auditory noise on visual choice reaction time. Acta Psychologica, 58, 45-62.

WHITAKER, L. A. (1982). Stimulus-response compatibility for left-right discriminations as a function of stimulus position. Journal of Experimental Psychology: Human Perception \& Performance, 8, 865-874.

\section{NOTE}

1. Gabriele Gratton, who reviewed a previous draft of this paper, suggested the possibility that the effect of monaural noise is facilitatory at relatively short latencies and inhibitory at longer latencies. Thus, at short response latencies (like those for congruent visual noise), this effect may be reduced or even reversed. Following this alternative explanation, whether the effect of monaural noise adds to or interacts with that of other manipulations depends mostly on response latency. A strong version of this interpretation would predict that the strength and direction of the effect of monaural noise covaries with mean RT level. Obviously, this prediction is not supported by the current data in that the conditions producing different RT levels (e.g., congruent vs. incongruent character arrays) show noise effects of equal magnitudes, whereas conditions with similar RT levels (e.g., blocked vs. mixed arrow arrays) show noise effects that are considerably different. However, a weaker version of the alternative explanation might suggest that the strength and direction of the monaural noise effect depends on the time at which location information associated with the ear stimulated becomes available vis-à-vis the processing of the visual stimulus. As Gratton pointed out, this version of the alternative explanation can be put to a test using a stimulus onset asynchrony (SOA) paradigm, in which the monaural noise is presented at different SOAs with respect to the visual stimulus. This test will be performed in future work that assesses the relation between monaural noise and visual information processing.

(Manuscript received August 27, 1987; revision accepted for publication December 9, 1987.) 